\title{
Exploring the mythical abscopal effect: Radiation and programmed cell death protein 1 (PD-1) blockade for hepatocellular carcinoma
}

\author{
Jun Won Kim and Ik Jae Lee \\ Department of Radiation Oncology, Yonsei University College of Medicine, Seoul, Korea
}

Keywords: Carcinoma, Hepatocellular; Programmed cell death 1 ligand 2 protein; Radiation; Radiobiology

\section{See Article on Page 144}

The term "abscopal" (ab-, a prefix meaning "position away from," and scopus [Latin] meaning "mark or target for shooting at") was first used by Mole in 1953 to describe the effects of ionizing radiation "at a distance from the irradiated volume but within the same organism." Radiotherapy (RT) induces immunogenic cell death, leading to the production and release of cytokines and chemokines in the tumor microenvironment, followed by infiltration of dendritic cells (DCs) into the tumor site. Activation of DCs, which act as antigen presenting cells, causes priming and expansion of tumor-reactive T-cells within the irradiated tumor and in the draining lymph nodes. These activated, tumor-specific T-cells in-turn migrate and eliminate non-irradiated tumors, hence resulting in the abscopal effect. ${ }^{2}$

The mechanism of abscopal effect has long remained poorly understood. In 2004, Demaria et al. ${ }^{3}$ suggested that the abscopal effect may be immune-mediated. Preclinical models have estab- lished that the abscopal effect is T-cell-dependent ${ }^{4}$ and results from radiation "priming" of the immune system. ${ }^{5}$ In recent years, the use of immune checkpoint inhibitors (ICls), such as ipilimumab or pembrolizumab, has greatly increased the prevalence of abscopal effects among selected patients, including those with metastatic melanoma. ${ }^{6,7}$

The mechanism underlying the increased abscopal effect following RT-ICI combination is unclear. In the current issue of Clinical and Molecular Hepatology, Yoo et al. ${ }^{8}$ conducted a preclinical study to investigate this issue using a murine syngeneic hepatocellular carcinoma (HCC) model. After transplanting Hepa 1-6 cells in both hind legs of immunocompetent C57BL/6 mice, they irradiated the tumors in primary sites and observed abscopal effects in the non-irradiated tumors, with or without anti-programmed cell death protein 1 (PD-1) antibodies. They reported significantly enhanced abscopal effect and increased infiltration of activated cytotoxic T-cells in both irradiated and non-irradiated tumors when combinatorial therapy with RT and anti-PD-1 antibodies was employed. Previous studies on combinatorial therapy

\begin{abstract}
Abbreviations:
cGAS, cyclic GMP-AMP synthase; CTLA-4, cytotoxic T-lymphocyte-associated protein 4; DCs, dendritic cells; HCC, hepatocellular carcinoma; ICls, immune checkpoint inhibitors; ICV, inferior vena cava; PD-1, programmed cell death protein 1; SBRT, stereotactic body radiotherapy; STING, stimulator of interferon genes; TDLNs, tumor-draining lymph nodes
\end{abstract}

\section{Corresponding author : Ik Jae Lee}

Department of Radiation Oncology, Gangnam Severance Hospital, Yonsei University College of Medicine, 211 Eonju-ro, Gangnam-gu, Seoul 06273, Korea

Tel: +82-2-2019-3152, Fax: +82-2-2019-4855

E-mail: ikjae412@yuhs.ac

https://orcid.org/0000-0001-7165-3373 
with RT and immunotherapy dealt with orthotopic HCC models, thus allowing the observation of effect of the combinatorial treatment on the primary tumor, though not necessarily the abscopal effect. ${ }^{9,10}$ In this context, Yoo et al. ${ }^{8}$ provides initial information on the impact of RT-immunotherapy combination on non-irradiated metastatic tumors using a murine HCC model. Many methods have been attempted to increase the prevalence of the abscopal effect, including the combination of RT with cytokines, stimulation of DCs to activate tumor antigen-presentation, vaccination with autologous tumor cells, targeting Toll-like receptors, and combination of RT and ICIs. ${ }^{2}$ Cytotoxic T-lymphocyte-associated protein 4 (CTLA-4) and anti-PD-1 are cell surface molecules which prevent T-cell activation or reinvigoration following chronic antigen exposure. Inhibiting these T-cell checkpoints leads to greater anti-tumor T-cell activity.

Yoo et al. ${ }^{8}$ also showed that $16 \mathrm{~Gy}$ in two fractions inhibited the growth of both irradiated and non-irradiated tumors more effectively with higher infiltration of cytotoxic T-cells compared to a single $8 \mathrm{~Gy}$ fraction. The authors concluded that these results indicated "the potential radiation dose-dependency of the abscopal effect." In recent years, the utilization of stereotactic body radiotherapy (SBRT) has increased, and there are suggestions that hypo-fractionated RT, which delivers higher doses of radiation per treatment or fraction, is associated with increased incidence of abscopal effects. While conventional RT is mainly directed at inducing apoptosis due to DNA damage, SBRT induces necrotic tumor cell death, which is a prerequisite for inducing an antitumor immune response. Yoo et al. ${ }^{8}$ explained that there is a window for the doses per fraction likely to induce abscopal effect, and thus their choice of the hypofractionated regimen. Using a similar experimental design, Dewan et al. ${ }^{11}$ tried to find the optimal RT dose and fractionation to induce the abscopal effect in combination with immunotherapy. They injected TSA mouse breast cancer cells into both hind legs of syngeneic mice, and irradiated the primary site with three distinct RT regimens ( $20 \mathrm{~Gy} \times 1,8 \mathrm{~Gy} \times 3$, or 6 Gy $\times 5$ fractions). As a result, they reported that fractionated RT, not single-fraction RT, induced immune-mediated abscopal effect when combined with CTLA-4 blockade." Later, they showed that the balance between cytosolic DNA and DNA exonuclease Trex1 activation plays a crucial role in the dose effect of RT-immunotherapy combination. RT induces accumulation of double-strand DNA in the cytosol, and cytosolic DNA activates the DNA sensor cyclic GMP-AMP synthase (cGAS) and its downstream effector, i.e., stimulator of interferon genes (STING). Activation of the cGAS/STING pathway results in interferon- $\beta$ secretion by cancer cells, leading to DC recruitment and activation, which are essential for $C D 8^{+}$T-cell priming and antitumor immunity. Dewan et al. ${ }^{11}$ showed that RT-induced cytosolic DNA accumulation is directly proportional to the fractional dose; however, doses above 12-18 Gy per fraction caused activation of DNA exonuclease Trex1 and degradation of cytosolic DNA, leading to attenuation of the RT-induced immunologic response. In this context, 8 Gy used by Yoo et al. ${ }^{8}$ falls in the range of optimal fractional doses for investigating the mechanism underlying the abscopal effect. ${ }^{12}$ Many questions remain to be answered regarding the optimization of RT-ICl combination, including the sequence of RT and ICIs (whether $\mathrm{ICl}$ can be administered before RT, after RT, or concurrently). There have been suggestions that anti-PD-L1 therapy is more effective with concurrent administration, while anti-CTLA-4 therapy is more effective when provided prior to RT; trials involving administration of multiple ICls with RT are currently underway. ${ }^{13}$

Yoo et al. ${ }^{8}$ showed that a higher-dose radiation also increased activated DC counts in the tumor-draining lymph nodes (TDLNs), which had a higher expression of PD-L1. While irradiation of the tumor site is capable of T-cell priming and activation, TDLNs with high tumor antigen load can provide a rich tumor drainage network and are important sites for DC-mediated antitumor T-cell stimulation, a critical step in T-cell activation with anti-PD-L1 therapy. ${ }^{14}$ Deng et al..$^{15}$ demonstrated that the STING signaling axis activated in DCs promotes an anti-tumor $\mathrm{CD}^{+}{ }^{+} \mathrm{T}$-cell response with an increased frequency of interferon- $\gamma^{+} \mathrm{CD} 8^{+} \mathrm{T}$-cells in TDLNs; and this phenomenon was also observed by Yoo et al. ${ }^{8}$ While these two studies did not involve the irradiation of TDLNS, Buchwald et al. ${ }^{14}$ injected modified B16F10 cells in bilateral flanks of C57BL/6 mice and irradiated either one flank tumor (10 Gy $\times 1$ ) and TDLNs ( $3 \mathrm{~Gy} \times 3$ ) or one flank tumor alone. They found that tumor irradiation stimulated the proliferation of total $C D 8^{+} T$-cells and stem-like CD8 ${ }^{+} T$-cells, a subset of CD8 ${ }^{+} T$-cells that rapidly proliferate following CD8 ${ }^{+} \mathrm{T}$-cell exhaustion by anti-PD-L1 therapy, in the TDLNs. In contrast, the irradiation of tumor and TDLNs resulted in a reduction in the abscopal effect as well as the number of total tumor-specific CD8 ${ }^{+}$T-cells and stem-like CD8 ${ }^{+} T$-cells in both the irradiated and unirradiated tumors. ${ }^{14}$ The role of TDLNs in mediating the abscopal effects—as investigated by Yoo et al. ${ }^{8}$ and others-is clinically significant and warrants further investigation, as the effect of RT-ICI combination on the abscopal effects may be compromised when patients are subjected to regional nodal irradiation or surgical removal of LNs.

Albeit rare, a number of cases of abscopal effects occurring in HCC patients have been reported in the literature. These cases in- 
clude palliative RT for bone metastasis resulting in the regression of primary $\mathrm{HCC}^{16,17}$ irradiation of inferior vena cava (IVC) invasion resulting in the regression of multiple intrahepatic metastases, ${ }^{18}$ irradiation of a metastatic mediastinal lymph node resulting in the regression of lung metastasis, ${ }^{19}$ and irradiation of primary HCC or IVC resulting in the regression of multiple lung or pleural metastases. ${ }^{20,21}$ Chino et al. ${ }^{5}$ reported an unusual case of SBRT for primary lung cancer resulting in complete remission of primary HCC. Although these clinical observations suggest the potential role of combining RT and ICls for metastatic HCCs, the RT-ICl combination should not be investigated for the sole purpose of inducing an abscopal effect, until we have more sufficient data to support the benefit of such a practice. Recent preclinical and retrospective studies have shown promising results for the combinatorial therapy with RT and ICls for advanced HCC., ${ }^{9,22}$ In this context, the study by Yoo et al. ${ }^{8}$ provides valuable insight into the mechanisms underlying enhanced abscopal effect of treating HCC with a combination of RT and ICls.

\section{Authors' contribution}

Jun Won Kim: manuscript writing and critical revision; Ik Jae Lee: critical revision and supervision

\section{Conflicts of Interest}

The authors have no conflicts to disclose.

\section{REFERENCES}

1. Mole RH. Whole body irradiation; radiobiology or medicine? $\mathrm{Br} J$ Radiol 1953;26:234-241.

2. Yilmaz MT, Elmali A, Yazici G. Abscopal effect, from myth to reality: from radiation oncologists' perspective. Cureus 2019;11:e3860.

3. Demaria S, Ng B, Devitt ML, Babb JS, Kawashima N, Liebes L, et al. Ionizing radiation inhibition of distant untreated tumors (abscopal effect) is immune mediated. Int I Radiat Oncol Biol Phys 2004; $58: 862-870$

4. Park SS, Dong H, Liu X, Harrington SM, Krco CJ, Grams MP, et al. PD-1 restrains radiotherapy-induced abscopal effect. Cancer Immunol Res 2015;3:610-619.

5. Chino F, Pollis KE, Choi S, Salama JK, Palta M. Stereotactic body radiation therapy-induced abscopal effect on hepatocellular carcinoma after treatment for lung cancer: a case report. Hepatology 2018;68:1653-1655.

6. Postow MA, Callahan MK, Barker CA, Yamada Y, Yuan J, Kitano S, et al. Immunologic correlates of the abscopal effect in a patient with melanoma. N Engl J Med 2012;366:925-931.

7. Hiniker SM, Reddy SA, Maecker HT, Subrahmanyam PB, RosenbergHasson $Y$, Swetter SM, et al. A prospective clinical trial combining radiation therapy with systemic immunotherapy in metastatic melanoma. Int J Radiat Oncol Biol Phys 2016;96:578-588.

8. Yoo GS, Ahn WG, Kim SY, Kang W, Choi C, Park HC. Radiationinduced abscopal effect and its enhancement by programmed cell death 1 blockade in the hepatocellular carcinoma: a murine model study. Clin Mol Hepatol 2021;27:144-156.

9. Friedman D, Baird JR, Young KH, Cottam B, Crittenden MR, Friedman $S$, et al. Programmed cell death-1 blockade enhances response to stereotactic radiation in an orthotopic murine model of hepatocellular carcinoma. Hepatol Res 2017;47:702-714.

10. Kim KJ, Kim JH, Lee SJ, Lee EJ, Shin EC, Seong J. Radiation improves antitumor effect of immune checkpoint inhibitor in murine hepatocellular carcinoma model. Oncotarget 2017;8:41242-41255.

11. Dewan MZ, Galloway AE, Kawashima N, Dewyngaert JK, Babb JS, Formenti SC, et al. Fractionated but not single-dose radiotherapy induces an immune-mediated abscopal effect when combined with anti-CTLA-4 antibody. Clin Cancer Res 2009;15:5379-5388.

12. Vanpouille-Box C, Alard A, Aryankalayil MJ, Sarfraz Y, Diamond JM, Schneider RJ, et al. DNA exonuclease Trex1 regulates radiotherapyinduced tumour immunogenicity. Nat Commun 2017;8:15618.

13. Choi C, Yoo GS, Cho WK, Park HC. Optimizing radiotherapy with immune checkpoint blockade in hepatocellular carcinoma. World J Gastroenterol 2019;25:2416-2429.

14. Buchwald ZS, Nasti TH, Lee J, Eberhardt CS, Wieland A, Im SJ, et al. Tumor-draining lymph node is important for a robust abscopal effect stimulated by radiotherapy. J Immunother Cancer 2020;8:e000867.

15. Deng L, Liang $H, X u M$, Yang $X$, Burnette $B$, Arina A, et al. STING dependent cytosolic DNA sensing promotes radiation-induced type I interferon-dependent antitumor immunity in immunogenic tumors. Immunity 2014;41:843-852.

16. Ohba K, Omagari K, Nakamura T, Ikuno N, Saeki S, Matsuo I, et al. Abscopal regression of hepatocellular carcinoma after radiotherapy for bone metastasis. Gut 1998;43:575-577.

17. Nam SW, Han JY, Kim JI, Park SH, Cho SH, Han NI, et al. Spontaneous regression of a large hepatocellular carcinoma with skull metastasis. J Gastroenterol Hepatol 2005;20:488-492.

18. Nakanishi M, Chuma M, Hige S, Asaka M. Abscopal effect on hepatocellular carcinoma. Am J Gastroenterol 2008;103:1320-1321.

19. Okuma K, Yamashita H, Niibe Y, Hayakawa K, Nakagawa K. Abscopal effect of radiation on lung metastases of hepatocellular carcinoma: a case report. J Med Case Rep 2011;5:111.

20. Lock M, Muinuddin A, Kocha WI, Dinniwell R, Rodrigues G, D'Souza D. Abscopal effects: case report and emerging opportunities. Cureus 2015;7:e344.

21. Ohmatsu K, Hashimoto Y, Kawanishi M, Ishii Y, Kono S, Kuribayashi 


\section{CLINCAL and MOLECULAR}

Volume_27 Number_1 January 2021

S, et al. Abscopal complete regression of hepatocellular carcinoma with multiple pleural metastases. Int Canc Conf J. 2020 Sep 25. doi: 10.1007/s13691-020-00446-y.

22. Kim HJ, Park S, Kim KJ, Seong J. Clinical significance of soluble pro- grammed cell death ligand-1 (sPD-L1) in hepatocellular carcinoma patients treated with radiotherapy. Radiother Oncol 2018;129:130135. 